\title{
DEELIG: A Deep Learning-based approach to predict protein-ligand binding affinity.
}

\author{
Asad Ahmed ${ }^{1 \uparrow}$, Bhavika Mam², ${ }^{2,3 \pi}$, Ramathan Sowdhamini ${ }^{2 *}$
}

1. National Institute of Technology, Warangal, Telangana, India

2. National Centre for Biological Sciences, Tata Institute for Fundamental Research, GKVK Campus, Bangalore, Karnataaka, India

3. The University of Trans-Disciplinary Health Sciences and Technology (TDU), Bangalore, Karnataka, India

\section{\ Co-first author \\ * Corresponding author:}

Email: mini@ncbs.res.in (RS)

\begin{abstract}
Protein-ligand binding prediction has extensive biological significance. Binding affinity helps in understanding the degree of protein-ligand interactions and has wide protein applications. Protein-ligand docking using virtual screening and molecular dynamic simulations are required to predict the binding affinity of a ligand to its cognate receptor. In order to perform such analyses, it requires intense computational power and it becomes impossible to cover the entire chemical space of small molecules. Recent developments using deep learning has enabled us to make sense of massive amounts of complex datasets where the ability of the model to "learn" intrinsic patterns in a complex plane of data is the strength of the approach. Here, we have incorporated Convolutional Neural Networks to find spatial relationships amongst data to help us predict affinity of binding of proteins in whole superfamilies towards a diverse set of ligands without the need of a docked pose or complex as input. The models were trained and validated using a detailed methodology for feature extraction. We have also tested DEELIG on protein complexes relevant to the current public health scenario. Our approach to network construction and training on protein-ligand dataset prepared in-house has yielded novel insights.
\end{abstract}




\section{Introduction}

Proteins are a diverse class of dynamic macromolecular structures in living organisms and are essential for the biochemistry and physiology of the organism. Depending on their functional role (s), proteins may bind to other proteins, peptides, nucleic acids and non-peptide ligands with varying affinities. Determining protein-ligand affinity helps in understanding the reaction mechanism and kinetics of the reaction, especially when experimental approaches may not be feasible, and has applications in drug development and pharmacology [1].

Protein-ligand interaction is measured in terms of Binding affinity. The stronger the readout for binding affinity, the stronger the interaction between protein and ligand may be inferred. It is quantified in terms of Inhibition constant $(\mathrm{Ki})$, dissociation constant $(\mathrm{Kd})$, changes in free energy measures (delta G, delta H and IC50) [2]. Predicting binding affinity between a protein and ligand complements experimental approaches and is usually used as a start-point for the latter. Classical prediction methods to score free binding energies of small ligands to biological macromolecules such as MM/GBSA and MM/PBSA typically rely on molecular dynamic simulations for calculations and aid in-silico docking and virtual screening as well as experimental approaches. However, there is a trade-off between computational resources and accuracy [3].

With a recent shift towards the use of machine learning and deep-learning based methods in the field of structural biology, making biologically significant predictions using regression and 'learning' intrinsic patterns in a complex plane of available data has led to resourceoptimal predictions without compromising on accuracy. Deep learning has been known to learn representations and patterns in complex data forms. Our aim was to apply deep learning to predict binding affinity of protein- non-peptide ligand interaction without the need of a docked pose as input.

Convolutional Neural Networks (CNN) are deep neural networks that use an input layer, output later as well as convolutional hidden layer(s). The first CNN was incorporated by LeCunn in 1998 [4] the connectivity pattern of which was inspired by the elegant experiments of Hubert and Weisel on the mammalian visual cortex in the 1960s [5]. With the growing technical advancements and massive amounts of data, CNNs have emerged popular in biological fields in the recent decade with various applications [6].

In our study, we have used CNNs to provide a quantitative estimate of protein-ligand binding using various sets of features corresponding to protein and ligand respectively by finding spatial relationships amongst the data without using docked poses as input. Our approach was validated using ligand-bound complexes from kinases superfamily in the PDB. Kinases belong to a class of enzymes required for substrate-dependent phosphorylation. They are represented across diverse cellular functions like signaling, differentiation, glycolysis [7]. We have also tested our model on COVID-19 main protease [8] of the novel coronavirus strain complexed with various inhibitors of which binding affinities have not been predicted or experimentally determined so far. 


\section{Materials and Method}

\section{Novel Dataset: Raw Data}

The raw data for our novel database was obtained from RCSB PDB (9) database, where following were selected as the query parameters.

- Chain Type: Protein Chain, No DNA or RNA or DNA/RNA Hybrid.

- Binding Affinity: Kd or Ki value present.

- Chemical Components: Has ligand (s)

- X-ray crystallography method: Resolution upto $2.5 \mathrm{~A}$.

These criteria resulted in a list of 5464 protein PDB IDs, 2568 complexed ligand (s) and corresponding binding affinity values. The search results include the structures present in PDBdatabase, PDBBind $(10,11,12)$, PDBMoad $(13,14)$ and $\operatorname{scPDB}(15)$ for its results. Initial raw data database created contained protein structures in PDB format, protein sequences in FASTA format, ligand in SDF format and binding affinity values of corresponding protein-ligand pairs for 5464 complexes.

\section{Dataset Refinement}

The PDB, FASTA and SDF files filtered were further processed to refine our novel dataset, as shown in Figure 1. Protein-ligand complexes were 5,464 in number and corresponded to 29,650 complex unique chain-ligand pairs. Binding affinity values were obtained from the RCSB database and protein chain-ligand pairs with corresponding binding affinity as 0 were discarded to reduce statistical errors. This narrowed down the total complexes to 4,750 protein-ligand pairs.

Pocket information was extracted from the protein using Ghecom (16) and converted to MOL2 format using Chimera (17), which narrowed our results to 4699 pocket-ligand pairs. It narrowed down the size of the dataset to 4286 pocket-ligand pairs.

We discarded other protein-ligand pairs with missing PSSM profiles, secondary structure or dihedral angle information.

It resulted in a total of 4041 pocket-ligand pairs, which corresponds to 7414 pocket-ligand pairs containing unique chains. 


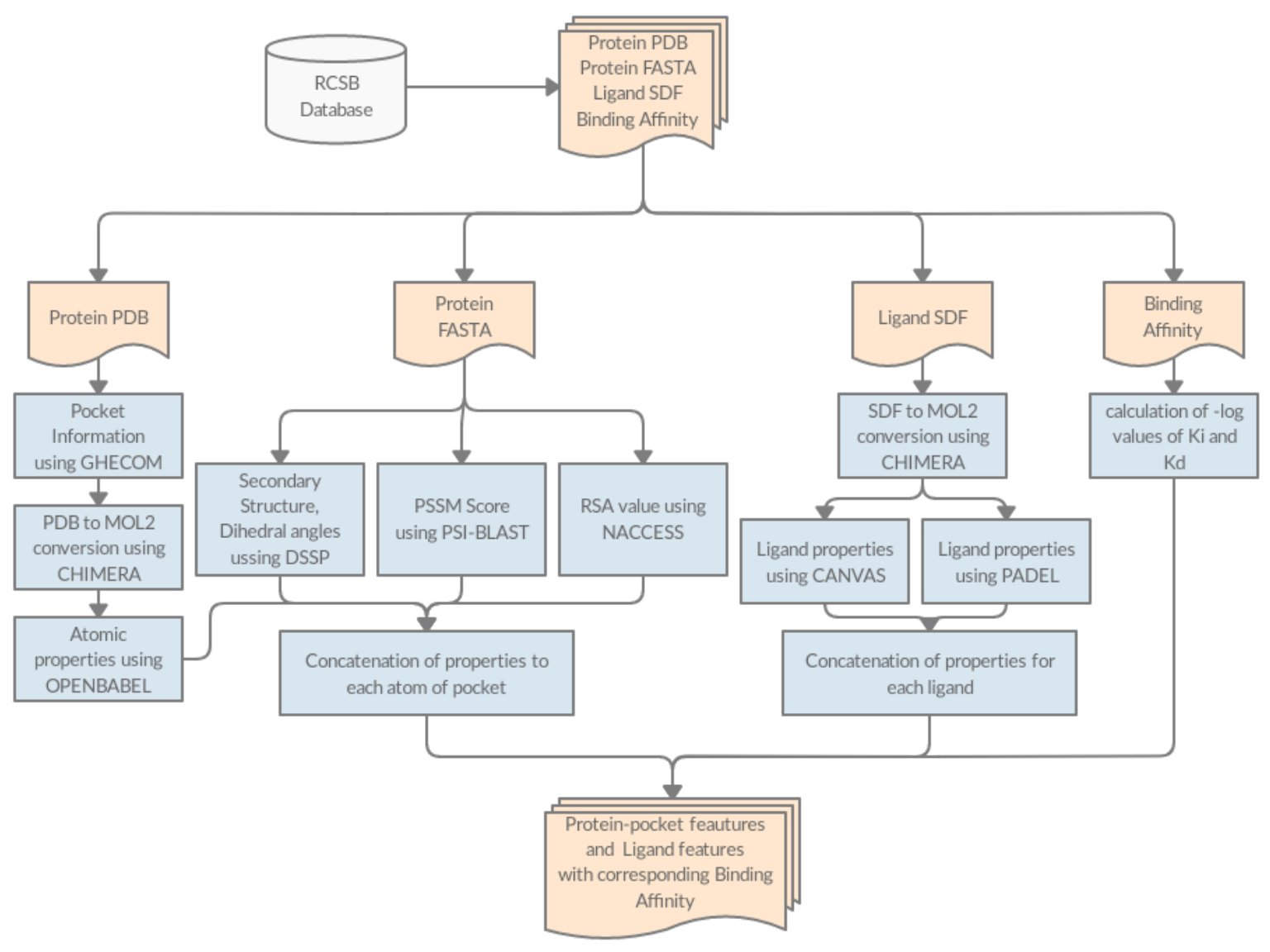

Figure 1: Feature Extraction pipeline

\section{Feature Extraction}

Training the deep learning network on raw information is known to result in longer time for convergence and less accuracy. We followed a conventional methodology for feature extraction and used the deep learning framework to learn the interaction between the protein-pocket and ligand for their affinity prediction.

\section{Protein-Pocket features}

A comprehensive two-level feature extraction methodology, one at the atomic level and the other at the level of amino acids utilizing structural information and protein sequence respectively.

\section{Atomic Level (19 Bits)}

- 9 Bit 1 hot or all null hot encoding for atom types: B, C, N, O, P, S, Se, halogen and metal.

- 1 integer for hybridization 
- 1 integer representing the number of bonds with heavy atoms

- 1 integer representing the number of bonds with hetero atoms

- 5 bits (1 if present) encoding properties defined with SMARTS patterns: hydrophobic, aromatic, acceptor, donor and ring

- 1 float for partial charges

- 1 integer to distinguish between ligand as -1 and protein as 1

\section{Amino Acid level (25 Bits)}

We utilized the sequence information of protein to get more features about the protein pocket-ligand interaction.

- Position-Specific Scoring Matrix (PSSM): PSSM is a matrix that represents the probability of mutation at each point of the sequence. It gives a 20 bit- probability for each amino acid at each location. PSSM profiles were obtained using PSI-BLAST (18) with SwissProt as subject database and E-value threshold as 0.001. Chains with less than 50 amino acids were removed from the input dataset.

- Relative Solvent Accessibility (RSA): It is encoded by 1 bit of information for each amino acid that provides whether it is buried or exposed to the solvent. We set a threshold of $25 \%$ in RSA values. RSA was obtained using NACCESS (19).

- Secondary Structure: It is encoded by 1 bit of information about the structure as coil, helix or plate and was predicted using the $\operatorname{DSSP}(20,21)$.

- Dihedral Angles: It is encoded by 2 bits of information with phi / psi angles of each of the amino acids and was predicted using $\operatorname{DSSP}(20,21)$ for obtaining dihedral angles.

\section{Ligand Features}

Standard ligand features were calculated for ligands in our dataset using PADEL (22) and 1D, 2D and chemical fingerprints, which includes hybridisation, atom pair interaction, counts of various functional group.

We also used QikProp (34) and QIKPROP (23) to derive ADMET (Absorption, Distribution, Metabolism, Excretion, and Toxicity) properties, which includes the physical properties, solubility and partition coefficients. The exhaustive list of every property calculated is given in the appendix.

It results in a $1 \mathrm{D}$ array of 14,716 dimensions containing the various properties of a given ligand. This is used as a feature vector representing the ligand represented in MOL2 format.

\section{Grid Formation}

The three-dimensional co-ordinates of atoms were converted into a $3 \mathrm{D}$ grid of resolution $10 \AA$ with $1 \AA$ spacing between the two axes centered along the centroid of the ligand. Atoms outside each such grid were discarded. The atoms lying inside the grid were rounded up to 
the nearest coordinate of the grid where features of corresponding atoms that lay in the same coordinates were added up.

This resulted in projecting ligand-interacting residues into a three-dimensional cube with features representing the atomic as well as protein-based properties of each atom of the protein pocket.

\section{Strategies}

Detailed and complete block diagrams with inputs are provided in Figures 2, 3 as well as in Supplementary Materials.

\section{Atomic Model}

\section{Preprocessing}

Features were calculated at the atomic level (Section 4.1.1) corresponding to each atom of an amino acid and ligand. A 19-bit vector was calculated that uniquely identified each of the atoms in the $3 \mathrm{D}$ co-ordinates of a given protein-pocket and ligand complex. A 4D tensor each of size $\mathrm{m} \times \mathrm{m} \times \mathrm{m} \times 19$, i.e. the 3 coordinates (x, y, z) and the features, where $\mathrm{m}$ represents the number of atoms present in a complex was constructed as the feature vector representing the given protein pocket-ligand.

The 4D vector contains the protein-pocket features and was converted to a 3D grid using grid featurization (Section 4.3). The 3D- featurized grid is essentially a 4D tensor, where the coordinates are approximated to the points on the grid.

The dataset is converted to vectors and is divided into training:validation:test sets in ratio 80:10:10. 


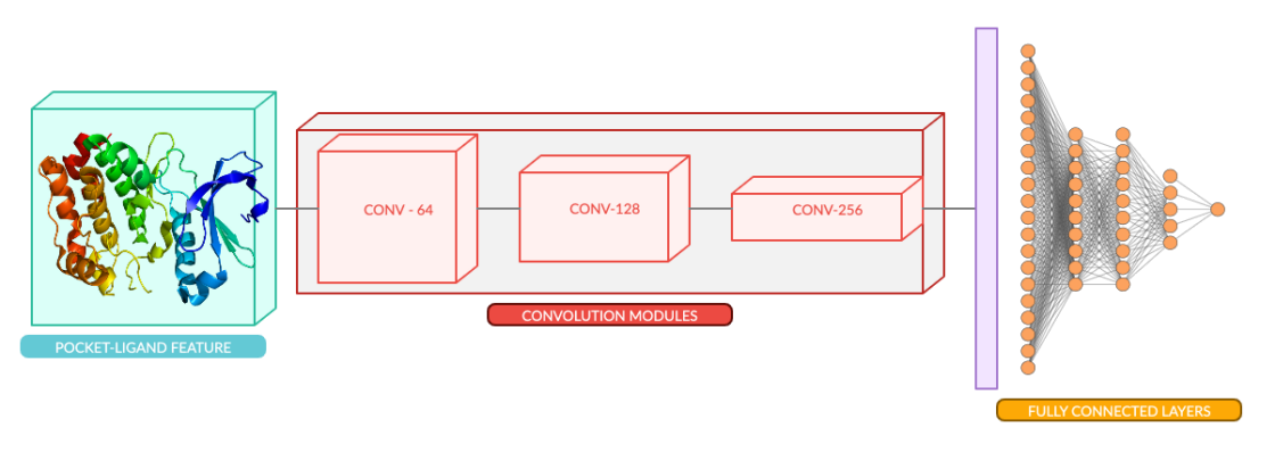

Figure 2: Training framework for Atomic Model. The framework is trained on 19 bits features each of protein-pocket and ligand together as input.

\section{Architecture}

Convolutional Neural Networks (24) have been used to capture spatial features in an image. We use CNNs to capture the interaction between ligand and protein atoms in threedimensional space. A network was constructed (Figure 2) with a 3D CNN of varying channel sizes of $[64,128,256]$ with non-linear activation ReLU after each layer, each 3D CNN had a filter of $5 \AA$ cube which was used to perform convolution operations. MaxPool (25) layer that acts in three dimensions to lower the dimension with a pool size of $2 \AA$ cube and Batch Normalization (26) layer is added after each CNN layer, this in turn decreases the training time and helps in faster convergence.

The latent features learnt from the above CNN layers were then flattened and used for calculating the binding affinity of the protein pocket-ligand pair. The CNN derives the relation among the 3D coordinates and their features, which would correspond well to the binding affinities of complexes.

The features from the last CNN layer are then flattened out, and passed through a fully connected neural network having the number of neurons as [1000, 500, 250] with ReLU as non-linearity after each layer. Dropout (25) is added after each layer to prevent overfitting by forcing the neural network to learn various other pathways by randomly assigning neurons to zero, 0.50 as Dropout threshold. Dense network predicts a regressive value of Binding Affinity, corresponding to a single neuron output.

Training framework is shown in Figure 2 and a detailed layer network is shown in Figure 4 (a). 


\section{Training}

The featurized protein-pocket grid formed was rotated to all 24 combinations possible, such that the network is able to learn in an orientation invariant form.

The network was trained by taking Mean Square error between the predicted and actual values as a loss function. The network was optimized using Adam (27) as the optimizer with a learning rate of 1e-5 and weight decay of 0.001 for 20 epochs. Network was trained on an Nvidia Pascal GPU using Pytorch (28) as the framework.

\section{Composite Model}

\section{Preprocessing}

Features were calculated at the amino acid level (Section 4.1.2) and were concatenated alongside the atomic level features (Section 4.1.1) to each atom of amino acid. It results in a 44-bit vector uniquely identifying each of the atoms in the 3D co-ordinates of a given protein. A 4D tensor each of sizes $\mathrm{m} \times \mathrm{m} \times \mathrm{m} \times 44$, i.e. the 3 coordinates $(\mathrm{x}, \mathrm{y}, \mathrm{z})$ and the features, where $m$ represents the number of atoms present in a complex is constructed as the feature vector of protein pocket.

The 4D vector contains the protein-pocket features, it was converted to a 3D grid using grid featurization (Section 4.3). The 3D featurized grid is essentially a 4D tensor, where the coordinates are approximated to the points on the grid.

The ligands were separately featurized by calculating the ligand properties (Section 4.2), which results in a $1 \mathrm{D}$ tensor.

The dataset is converted to vectors and is divided into training:validation:test sets in ratio 80:10:10. 


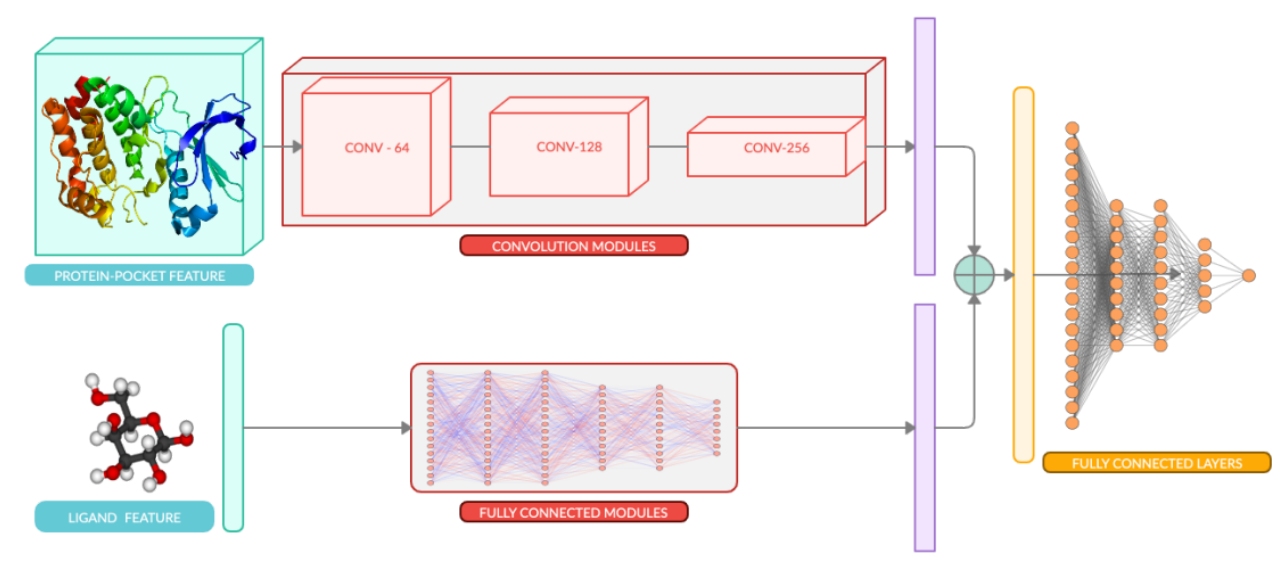

Figure 3: Training framework for Composite Model. The framework is trained on 44 bits features of protein-pocket and 14716 bits of ligand as separate inputs.

\section{$\underline{\text { Architecture }}$}

A multi-input network was constructed (29) with a 3D CNN (24) of varying channel sizes of $[64,128,256]$ with non-linear activation ReLU after each layer, each 3D CNN had a filter of $5 \AA$ cube which was used to perform convolution operations. We also added MaxPool (25) layer that acts three-dimensionally to lower dimensionality while retraining features learnt after each CNN layer. It has a filter size of $2 \AA$ cube. Batch Normalization (26) layer was added after each CNN module for faster convergence.

The ligand features were passed through the dense layers of sizes [7000, 5000, 2000] with ReLU as non-linearity after each layer and we also perform dropout operations after each dense layer to prevent it from overfitting (30). This results in a latent vector representing the relevant features for each ligand.

The latent output from the CNN layers is flattened and concatenated with the latent feature vector of ligand, to create one single feature vector of protein pocket-ligand interactions. This vector is passed through a densely connected neural network having the number of neurons as [7000, 2000, 500, 200] with ReLU as non-linearity after each layer and we used Dropout after each layer also to prevent overfitting forcing the neural network to learn various other pathways by randomly assigning weights of neurons to zero, with 0.50 as Dropout threshold. This dense network finally predicts a regressive value of Binding Affinity, corresponding to a single neuron output.

Training framework is shown in Figure 3 and a detailed layer network is shown in Figure 4 (b) 


\section{Additional Case studies of specific protein families}

Recently deposited complexes of COVID-19 main protease with various inhibitors deposited in the PDB were used for the purpose of our study (Table 3). The crystal structure complexes (PDB IDs: 5R7Y, 5R7Z, 5R82, 5R84) of the COVID-19 main protease with inhibitors ( (Z45617795: N-[ (5-methylisoxazol-3-yl)carbonyl]alanyl-L-valyl-N 1 - ( (1R,2Z)-4(benzyloxy)-4-oxo-1-\{[ (3R)-2-oxopyrrolidin-3-yl]methyl)but-2-enyl)-L-leucinamide); Z1220452176: ( \{N)-[2- (5-fluoranyl-1 \{H)-indol-3-yl)ethyl]ethanamide); Z219104216: 6(ethylamino)pyridine-3-carbonitrile; Z31792168:

2-cyclohexyl- \{N)-pyridin-3-yl-ethanamide)) respectively has been recently deposited in PDB (2020; unpublished).

Another study has deposited the complex of the COVID-19 main protease with a broad-spectrum inhibitor X77 (N- (4-tert-butylphenyl)-N-[(1R)-2-

(cyclohexylamino)-2-oxo-1- (pyridin-3-yl) ethyl]-1H-imidazole-4-carboxamide) (2020; unpublished).

In order to compare affinity of deoxycholate with homologous proteins of the periplasmic C-type cytochrome (Table 4), Ppc homologs PpcA (PDB: 1OS6), PpcB (PDB: 3BXU), PpcC (PDB: 3H33), PpcD (PDB: 3H4N) and PpcE (PDB: 3H34) and ligand deoxycholic acid (Pubchem CID: 222528) were gathered. These were processed and DEELIG was used to predict the binding affinity of each homolog with the ligand.

\section{Training}

The featurized protein-pocket grid formed was rotated to all 24 combinations possible, such that the network is able to learn in an orientation invariant form.

The featurized protein pocket-ligand pair of training set was passed through corresponding the network and trained by taking Mean Square error between the predicted and actual values as a loss function. The network was optimized using Adam (27) as the optimizer with a learning rate of 1e-5 and weight decay of 0.001 . The network was trained on an Nvidia Pascal GPU using Pytorch (28) as the framework.

\section{Performance Evaluation}

The predicted value of our regression-based approach is the negative natural logarithmic value of $\mathrm{Kd}$ or $\mathrm{Ki}$. This is then converted to its antilog to obtain $\mathrm{Kd}$ or $\mathrm{Ki}$ value in nanoMolar quantity.

The performance of the models was quantified using Mean Absolute error (MAE) and Root mean square error (RMSE). It was tested on validation and testing sets which were initially divided from our dataset as mentioned in the training section. Lower error corresponds to better learning capacity of the model. Standard deviation among the real and predicted values was also calculated.

The MAE, RMSE and SD values are shown in Table 1. 
Table 1: Predictions accuracy on test set of our novel dataset

\begin{tabular}{|l|l|l|l|}
\hline Method & MAE & RMSE & SD \\
\hline Atomic Model & 2.84 & 3.93 & 2.62 \\
\hline Composite Model & $\mathbf{2 . 2 7}$ & $\mathbf{3 . 0 7}$ & $\mathbf{2 . 0 6}$ \\
\hline
\end{tabular}

For the purpose of training and testing models, one NVIDIA Tesla P100 GPU cluster was used. Computational time taken for featurization of the dataset, training and testing were 52 hours, 22 hours and 8 minutes respectively.

\section{Results and Discussion}

Two modules were trained. The first module was trained using a small set of features for protein and ligand, which were represented together in a 3D grid space. This approach has also been part of a previous study (29). However, the previous study uses a restricted ligand set that does not involve larger ligands. Here we have used a diverse set of ligands as one of our inputs. With training of Atomic Model for 35 epochs, MAE score of 2.84 was achieved (Table 1).

We constructed another module that enabled us to improve on the ligand and protein based information. To this purpose, we used an increased feature vector size which amounted to 14716 bits in size for ligand and 44 bits for each atom of protein. With training of Composite Model for only 4 epochs, MAE score of 2.27 was achieved (Table 1).

The performance of our model was further evaluated using ligand-bound complexes from the kinase superfamily from PDB. The composite model outperformed the atomic model significantly and with lower standard deviation. (Table 2).

Table 2 : Predictions accuracy on kinases

\begin{tabular}{|l|l|l|l|}
\hline Method & MAE & RMSE & SD \\
\hline Atomic Model & 2.48 & 3.24 & 3.11 \\
\hline Composite Model & $\mathbf{2 . 2 4}$ & $\mathbf{2 . 7 1}$ & $\mathbf{2 . 6 7}$ \\
\hline
\end{tabular}

In light of the ongoing coronavirus pandemic, we tested protein-ligand complexes from the coronavirus (CoV) family. The COVID-19 main protease is a key enzyme for the novel strain of coronavirus that is being implicated in the pandemic. A recent study involved testing of in-vitro binding efficacy of coronavirus COVID-19 virus main protease (Mpro) with a potent reversible synthetic inhibitor, N3 (31). However, the highly 
potent inhibition by $\mathrm{N} 3$ rendered the experimental determination of binding affinity not achievable. Using the structure of Mpro at high resolution (7BQY: 1.7 Angstrom), we have been able to predict the binding affinity of $\mathrm{N} 3$ to $3.1 \mathrm{e}+4$ nanomolar (Table 3 ). This value agrees with the observed high affinity in the course of recent experiments (31).

We used complexes of COVID-19 main protease with various inhibitors (Materials and Methods; Table 3) to predict their respective binding affinities as their experimental values have not been made available. Based on our model-based predictions, broad spectrum inhibitor X77 scores for highest affinity followed by ligands Z45617795, N3, Z31792168, Z1220452176 and Z219104216 in the order of decreasing binding affinity (Table 3) strengthening the suitability of X77 as a potential candidate against COVID-19 virus protease

Table3: Predictions of Binding Affinity on COVID-19 complexes

\begin{tabular}{|l|l|l|l|}
\hline PDB & Ligand & - Log $(\mathbf{K d} / \mathbf{K i})$ & {$[$ Kd] or $[\mathbf{K i}]$ (nM) } \\
\hline $5 R 7 Y$ & Z45617795 & 11.96 & $6.39 \mathrm{e}+3$ \\
\hline $5 R 7 Z$ & Z1220452176 & 7.69 & $4.57 \mathrm{e}+5$ \\
\hline $5 R 82$ & Z219104216 & 6.12 & $2.18 \mathrm{e}+6$ \\
\hline $5 R 84$ & Z31792168 & 8.32 & $2.43 \mathrm{e}+5$ \\
\hline $6 \mathrm{~W} 63$ & $\mathrm{X} 77$ & 15.34 & $2.17 \mathrm{e}+2$ \\
\hline $7 B Q Y$ & $\mathrm{~N} 3$ & 10.38 & $3.10 \mathrm{e}+4$ \\
\hline
\end{tabular}

A triheme cytochrome from the sulfur-, metal- and radionuclide-reducing bacteria, Geobacter sulfurreducens, named PpcA binds strongly to deoxycholate [10]. However, its triheme paralogous counterparts $\mathrm{PpcB}, \mathrm{PpcC}, \mathrm{PpcD}$ and $\mathrm{PpcE}$ do not bind to deoxycholate $[11,12]$. Our results also predict that ligand deoxycholate binds with high affinity to periplasmic C-type cytochrome A (PpcA) but not to its homologs PpcB, PpcC, PpcD and PpcE (Table 4).

Table 4: Predictions of Binding Affinity on homologs of Periplasmic C-type Cytochrome (Ppc) family

\begin{tabular}{|c|c|c|}
\hline Homolog & PDB ID & $\begin{array}{c}\text { Prediction } \\
\text { Kd or Ki (uM) }\end{array}$ \\
\hline PpcA & 1 OS6 & 4.512 \\
\hline PpcB & $3 \mathrm{BXU}$ & 416.042 \\
\hline PpcC & $3 \mathrm{H} 33$ & 835.232 \\
\hline
\end{tabular}




\begin{tabular}{|l|l|l|}
\hline PpcD & $3 H 4 N$ & 483.678 \\
\hline PpcE & $3 H 34$ & 187.157 \\
\hline
\end{tabular}

\section{Conclusion}

Deep-learning based approaches have been implemented for prediction of binding affinity. One of the studies used atomic level features of complex in a CNN based framework for binding affinity prediction (35), while another study used protein sequence level features in a $\mathrm{CNN}$ based framework for prediction (36). Another approach used as been to use feature learning along with gradient boosting algorithms to predict binding affinity (36). Here, we provide a composite model that incorporates tripartite structural, sequence and atomic level features with those of the atomic and other chemical features of the ligand to predict binding affinity of a putative complex.

We propose a deep-learning based approach to predict ligand (eg., drug)-target binding affinity using only structures of target protein (PDB format) and ligand (SDF format) as inputs. Convolutional Neural Networks (CNN) were used to learn representations from the features extracted from these inputs and hidden layers in the affinity prediction task. We used two approaches to feature extraction- atomic level as well as composite level and compared their performance using the same network. We have trained on complexes from PDB across all taxa filtered as per few starting criteria including crystal quality. Our results are validated and reflected in the performance scores. The baseline to the results of our approach is the study by Stepniewska-Dziubinska et al 2018 [27], the performance of which our study has exceeded (Results).

Our algorithm relies on certain inputs including sensitive binding cavity detection by the Ghecom algorithm (Kawabata, 2010) that uses mathematical morphology to find both deep and shallow pockets (if any) in a given protein. The coordinates of the predicted binding cavity of the protein (grid) are rotated to various combinations and are placed around the centroid of the ligand and the resultant 4-D tensor is processed further for features along the CNN (Materials and Method). Hence, ligand-bound poses are not used as input. Our dataset has $\sim 5 \mathrm{k}+$ complexes and also includes complexes that were not part of PDBBind (which is usually used to benchmark and is derived from PDB). The ligand set we have used also represents a diverse set (Supplementary Materials SM Files 1 and 2) and is one of the highlights of our approach. The predictions from DEELIG can in fact help existing databases like RSCB PDB, PDBMoad and PDBBind in filling missing binding affinity data for complexes. 
We have constructed a novel dataset that represents a diverse set of ligands and using a novel deep learning based approach we have achieved significant improvement in prediction of binding affinity of protein-ligand complexes. Interestingly, our approach performed better without ligand coordinates as input. To counter filtering or noise reduction in our dataset, our dataset constructed is smaller than PDBBind (35) but we have overcome the constraints on ligand selection part of a previous study (29). Although our dataset contains 5464 complexes compared to 16,151 complexes found in PDBBind, the ligands used as part of our training include 452 unique ligands absent in PDBBind. This helps in achieving ligand diversity during training the $\mathrm{CNN}$ model. The similarity matrix constructed from the binary fingerprints of ligands used in the dataset supports our claim of improved ligand diversity in our dataset (Supplementary File S1).

We have highlighted a few examples such as complexes of kinases and viral drug targets only to reinforce the broader applicability of our approach (Tables 2 and 3). Our predictions are in line with experimental observations $[32,33,34]$ that deoxycholate binds to PpcA cytochrome but not to homologs PpcB - E cytochrome (Table 4).

We have also eliminated the need of providing ligands in a complex form with protein. Thus a given protein pocket may be tested for the degree of binding for any given ligand. This can be extended to predicting potential binding partners for proteins in other superfamilies as well. It is also important to consider that docking score and pose is not a reliable correlation with MM/GBSA poses (37). DEELIG can be used for a member of any protein superfamily and a non-peptide ligand, the docking pose of which may or may not be known.

The code repository for the project is publicly available at :

https://github.com/asadahmedtech/DEELIG

\section{Future Direction}

Binding affinity predictions through DEELIG can be extended to protein-ligand complexes of protein superfamilies where the affinity is quantitatively unknown due to experimental limitations or where the potential for binding is yet to be explored in vitro. A webserver to implement DEELIG for easy online access would be useful for the general scientific community and this will also be in the pipeline. A later version of DEELIG which is trained on peptide ligand dataset will also be worked on.

\section{References}

[1] Ahmed Aqeel, Smith Richard D, Clark Jordan J, Dunbar Jr. James B, and Carlson Heather A. Recent improvements to binding moad: a resource for protein ligand binding affinities and structures. Nucl. Acids Res., 43 (D):465-469, 2014. 
[2] Stephen F Altschul, Thomas L Madden, Alejandro A Schäffer, Jinghui Zhang, Zheng Zhang, Webb Miller, and David J Lipman. Gapped blast and psi-blast: a new generation of protein database search programs. Nucleic acids research, 25 (17):3389-3402, 1997.

[3] Jianxin Duan, Steven L Dixon, Jeffrey F Lowrie, and Woody Sherman. Analysis and comparison of $2 \mathrm{~d}$ fingerprints: insights into database screening performance using eight fingerprint methods. Journal of Molecular Graphics and Modelling, 29 (2):157-170, 2010.

[4] Samuel Genheden and Ulf Ryde. The $\mathrm{mm} / \mathrm{pbsa}$ and $\mathrm{mm} / \mathrm{gbsa}$ methods to estimate ligand-binding affinities Expert opinion on drug discovery, 10 (5):449-461, 2015.

[5] Tong He, Marten Heidemeyer, Fuqiang Ban, Artem Cherkasov, and Martin Ester. Simboost: a read-across approach for predicting drug-target binding affinities using gradient boosting machines. Journal of cheminformatics, 9 (1):1-14, 2017.

[6] Simon J Hubbard and Janet M Thornton. Naccess. Computer Program, Department of Biochemistry and Molecular Biology, University College London. 2 (1), 1993.

[7] David H Hubel and Torsten N Wiesel. Receptive fields, binocular interaction and functional architecture in the cat's visual cortex. The Journal of Physiology, 160 (1):106-154, 1962.

[8] Sergey Ioffe and Christian Szegedy. Batch normalization: Accelerating deep network training by reducing internal covariate shift. arXiv preprint arXiv:1502.03167, 2015.

[9] Jin, Z., Du, X., Xu,Y. et al. Structure of $\mathrm{M}^{\text {pro }}$ from SARS-CoV-2 and discovery of its inhibitors. Nature 582, 289-293 (2020)

[10] Robbie P Joosten, Tim AH Te Beek, Elmar Krieger, Maarten L Hekkelman, Rob WW Hooft, Reinhard Schneider, Chris Sander, and Gert Vriend. A series of pdb related databases for everyday needs. Nucleic acids research, 39 (suppl_1):D411-D419, 2010.

[11] Desaphy Jérémy, Bret Guillaume, Rognan Didier, and Kellenberger Esther. sc-pdb: a 3d-database of ligandable binding sites-10 years on. Nucleic Acids Research, 43 (D1):399-404, 2015.

[12] Wolfgang Kabsch and Christian Sander. Dictionary of protein secondary structure: pattern recognition of hydrogen-bonded and geometrical features. Biopolymers: Original Research on Biomolecules, 22 (12):2577-2637, 1983.

[13] Panagiotis L Kastritis and Alexandre MJJ Bonvin. On the binding affinity of macromolecular interactions: daring to ask why proteins interact. Journal of The Royal Society Interface, 10 (79):20120835, 2013.8

[14] Takeshi Kawabata. Detection of multiscale pockets on protein surfaces using mathematical morphology. Proteins: Structure, Function, and Bioinformatics , 78 (5):1195-1211, 2010.

[15] Diederik P Kingma and Jimmy Ba. Adam: A method for stochastic optimization. arXiv preprint arXiv:1412.6980,2014.

[16] Alex Krizhevsky, Ilya Sutskever, and Geoffrey E Hinton. Imagenet classification with deep convolutional neural networks. In Advances in neural information processing systems, pages 1097-1105, 2012. 
[17] Hu L and RD Smith MG Lerner HA Carlson ML, Benson. Binding moad (mother of all databases. Proteins, 60:333-40, 2005.

[18] Yann LeCun, Léon Bottou, Yoshua Bengio, and Patrick Haffner. Gradient-based learning applied to document recognition. Proceedings of the IEEE, 86 (11):2278-2324, 1998.

[19] Yanjun Li, Mohammad A Rezaei, Chenglong Li, Xiaolin Li, and Dapeng Wu.

Deepatom: A framework for protein-ligand binding affinity prediction. arXiv preprint arXiv:1912.00318, 2019.

[20] Seonwoo Min, Byunghan Lee, and Sungroh Yoon. Deep learning in bioinformatics. Briefings in bioinformatics, 18 (5):851-869, 2017.

[21] Benson ML, Smith RD, Khazanov NA, Dimcheff B, Beaver J, Dresslar P, Nerothin J, and Carlson HA. Bindingmoad, a high-quality protein-ligand database. Nucleic Acids Research, 36 (D):674-678, 2008.

[22] Hakime Öztürk, Arzucan Özgür, and Elif Ozkirimli. Deepdta: deep drug-target binding affinity prediction. Bioinformatics, 34 (17):i821-i829, 2018.

[23] Adam Paszke, Sam Gross, Soumith Chintala, and Gregory Chanan. Pytorch: Tensors and dynamic neural networks in python with strong gpu acceleration. PyTorch: Tensors and dynamic neural networks in Python with strong GPU acceleration, 6, 2017.

[24] Eric F Pettersen, Thomas D Goddard, Conrad C Huang, Gregory S Couch, Daniel M Greenblatt, Elaine C Meng, and Thomas E Ferrin. Ucsf chimera-a visualization system for exploratory research and analysis. Journal of computational chemistry, 25 (13):1605-1612, 2004.

[25] Peter W Rose, Bojan Beran, Chunxiao Bi, Wolfgang F Bluhm, Dimitris Dimitropoulos, David S Goodsell, Andreas Prlic, Martha Quesada, Gregory B Quinn, John D Westbrook, et al. The rcsb protein data bank: redesigned website and web services. Nucleic acids research, 39 (suppl_1):D392-D401, 2010.

[26] Karen Simonyan and Andrew Zisserman. Very deep convolutional networks for large-scale image recognition. arXiv preprint arXiv:1409.1556, 2014.

[27] Marta M Stepniewska-Dziubinska, Piotr Zielenkiewicz, and Pawel Siedlecki.

Development and evaluation of a deep learning model for protein-ligand binding affinity prediction. Bioinformatics, 34 (21):3666-3674, 052018.

[28] R Wang, X Fang, Y Lu, and S Wang. The pdbbind database: collection of binding affinities for protein-ligand complexes with known three-dimensional structures.J. Med. Chem., 47 (12):2977-80, 2004.

[29] R Wang, X Fang, Y Lu, CY Yang, and S Wang. The pdbbind database: methodologies and updates. J. MedChem., 48 (12):4111-9, 2005.

[30] Renxiao Wang, Xueliang Fang, Yipin Lu, Chao-Yie Yang, and Shaomeng Wang. The pdbbind database: methodologies and updates. Journal of medicinal chemistry, 48

(12):4111-4119, 2005.

[31] Chun Wei Yap. Padel-descriptor: An open source software to calculate molecular descriptors and fingerprints.Journal of computational chemistry, 32 (7):1466-1474, 2011 
[32] Pokkuluri PR, Londer YY, Duke NE, Long C, and Schiffer M. Family of Cytochrome c7-Type Proteins from Geobacter sulfurreducens: Structure of One Cytochrome c7 at 1.45 Å Resolution. Biochemistry, 2004, 43 (4), 849-859. DOI: 10.1021/bi0301439

[33] Pokkuluri PR, Londer YY, Yang X, et al. Structural characterization of a family of cytochromes c(7) involved in Fe(III) respiration by Geobacter sulfurreducens. Biochimica et Biophysica Acta. 2010 Feb:1797(2):222-232. DOI: 10.1016/j.bbabio.2009.10.007.

[34] Pokkuluri PR, Londer YY, Duke NE, Pessanha M, Yang X, Orshonsky V, Orshonsky L, Erickson J, Zagyanskiy Y, Salgueiro CA, Schiffer M. Structure of a novel dodecaheme cytochrome c from Geobacter sulfurreducens reveals an extended $12 \mathrm{~nm}$ protein with interacting hemes. J Struct Biol. 2011 Apr;174(1):223-33. doi: 10.1016/j.jsb.2010.11.022.

[35] Liu Zhihai, Li Yan, Han Li, Li Jie, Liu Jie, Zhao Zhixiong, Nie Wei, Liu Yuchen, and Wang Renxiao. Pdb-wide collection of binding data: current status of the pdbbind database. Bioinformatics, 31 (3):405-12, 2014.

[36] Binding of nicotinoids and the related compounds to the insect nicotinic acetyicholine receptor. Journal of Pesticide Science, 17 (4):231-236, 1992.

[37] Rastelli G, Del Rio A, Degliesposti G, Sgobba M. Fast and accurate predictions of binding free energies using MM-PBSA and MM-GBSA. J Comput Chem., 2010, 31(4):797-810. DOI: $10.1002 /$ jcc.21372.

[38] Schrödinger Release 2020-3: QikProp, Schrödinger, LLC, New York, NY, 2020

\section{Supplementary Files}

a. SM compressed folder: dataset.gz (can be retrieved from https://rive.google.com/file/d/1JE3gQuTXprRVghygAwR9HESvABHKED0L/view ?usp=sharing)

b. SM Files for Ligand diversity analysis: Similarity matrix (SM File 1) and clustering (SM File 2) of unique ligands-

https://drive.google.com/drive/folders/1Ar64qn8vD0sSdPWptPgOkPeM7pWghKi7?u $\underline{\mathrm{sp}}=$ sharing

c. SM File 3: Dataset_distribution.xls

d. SM File 4: Dataset_details.xls 


\section{Appendix}

\section{A.1: Property list for ligand features}

Following properties of ligand were calculated using PADEL (22),

- Basic Group Count

- Carbon Type

- Hybridization Ratio

- Manhold LogP (The Ratio of carbon to hetero atoms)

- Number of Aromatic bonds

- MACCSS Key

- Klehotaroth fingerprints (Types and Counts)

- AtomPair2D fingerprints (Types and Counts)

Following are ADMET and present in PADEL

- donorHB

- $a c c p t H B$

- Constitutional (Electronegativity)

- rotatableBondCounts (\#ringatoms)

- RuleofFive

- $V A B C$ (Volume)

- Weight (mol_MW)

Following $\{A D M E T)$ properties of ligand were calculated using QikProp (34) and QIKPROP $(16,21)$,

- Amine

- Amidine

- Acid

- Amide

- Rotor

- rtvFG (reactive functional groups)

- mol_MW, dipole

- Volume

- donorHB

- accptHB

- QPpolrz (polarizability) 
- SASA (probe of $1.4 \mathrm{~A}$ )

- FOSA (hydrophobic component of SASA)

- FISA (hydrophilic component of SASA)

- PISA (pi of SASA)

- WPSA (polar of SASA)

- SAFluorine

- SAamideO

- Partition coefficients $=>$
○ QPlogPC16
○ QPlogPoct
- QPlogPw
- $\mathrm{QP} \log \mathrm{Po} / \mathrm{w}$

- CIQPlogS (Conformation indie aqueous solubility)

- IP (ev) ( ionization potential)

- $\mathrm{EA}(\mathrm{eV})$ (electron affinity)

- \#metab (likely metabolic reactions)

- PSA (van der waals SA of polar N and O atoms)

- \#NandO, \#ringatoms (number of atoms in rings)

- \#in34 (number of atoms in 3 or 4 membered rings)

- \#in56 (number of atoms in 5 or 6 membered rings)

- \#noncon (ring atoms cannot form conjugated aromatic bonds)

- \#nonHatm (heavy atoms- nonhydrogen atoms)

- RuleOfThree

- RuleOfFive (lipinski violations)

- QPlogKhsa (binding to human serum albumin)

- PercentHuman-OralAbsorption

- Globular nature index 
bioRxiv preprint doi: https://doi.org/10.1101/2020.09.28.316224; this version posted January 3, 2021. The copyright holder for this preprint

(which was not certified by peer review) is the author/funder, who has granted bioRxiv a license to display the preprint in perpetuity. It is made available under aCC-BY-ND 4.0 International license.

\section{A.2 Network Layout for modules}

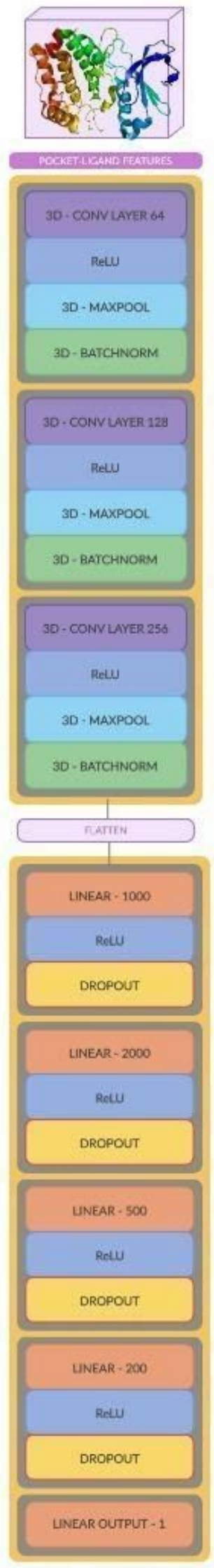

(a) Module 1 network

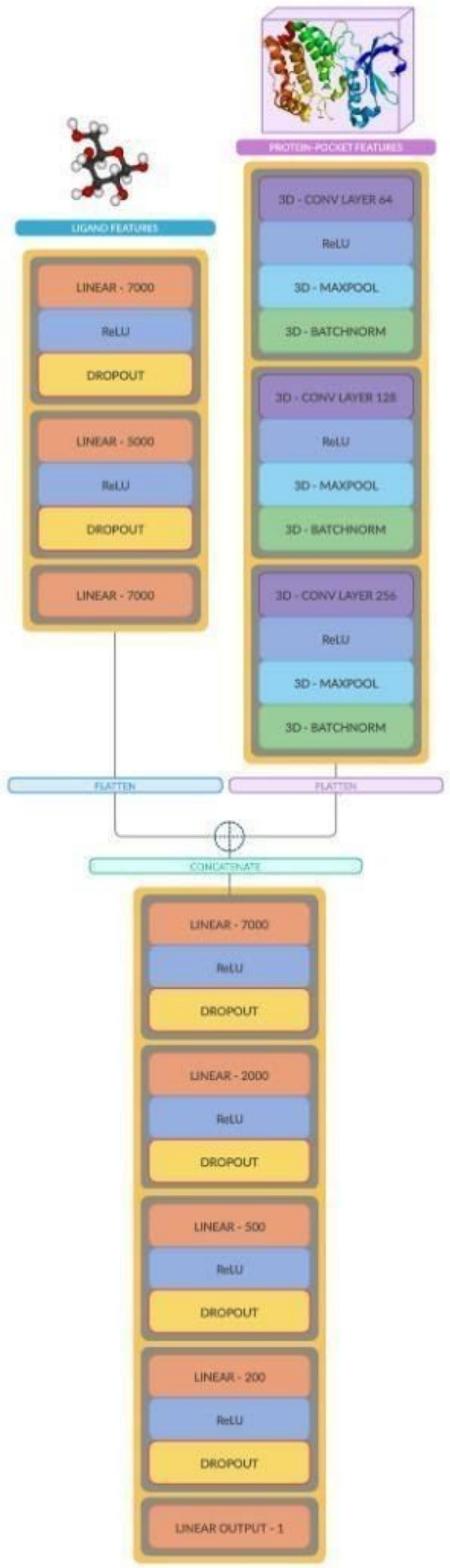

(b) Module 3 network 


\section{B.1 Kinases prediction dataset}

\begin{tabular}{|c|c|c|c|}
\hline PDBID & $\begin{array}{l}\text { GroundTruth }(-\log \\
(\mathrm{kd} / \mathrm{ki}))\end{array}$ & Predicted $(-\log (\mathrm{kd} / \mathrm{ki}))$ & Set \\
\hline 1ATP_ATP_E_355 & 14.33 & 10.162511 & training \\
\hline 1B38_ATP_A_381 & 12.89 & 11.011607 & training \\
\hline 1B39_ATP_A_381 & 13.64 & 10.672279 & training \\
\hline 1BX6_BA1_A_351 & 16.88 & 17.481277 & training \\
\hline 1KV1_BMU_A_391 & 11.34 & 11.811321 & training \\
\hline 1PXJ_CK2_A_500 & 8.11 & 8.140871 & training \\
\hline 1Q8T_Y27_A_930 & 9.22 & 8.202146 & training \\
\hline 1Q8U_H52_A_961 & 12.6 & 14.963462 & training \\
\hline 1Q8U_H52_A_962 & 12.6 & 12.837863 & training \\
\hline 1Q8W_M77_A_960 & 11.05 & 13.436903 & training \\
\hline 1R0E_DFN_A_702 & 20.73 & 18.322815 & training \\
\hline 1R0E_DFN_B_501 & 20.73 & 20.813835 & training \\
\hline 1TVO_FRZ_A_1001 & 11.68 & 14.313412 & training \\
\hline 1UNL_RRC_A_1293 & 3.97 & 5.2938104 & training \\
\hline 1UU3_LY4_A_1374 & 4.97 & 12.5554905 & training \\
\hline 1XH4_R69_A_351 & 14.9 & 10.410368 & training \\
\hline 1XH5_R68_A_1001 & 10.29 & 11.608167 & training \\
\hline 1XWS_BI1_A_1001 & 16.12 & 15.265529 & training \\
\hline 1YDT_IQB_E_351 & 14.51 & 11.634923 & training \\
\hline 2BAK_AQZ_A_401 & 14.81 & 13.099378 & training \\
\hline 2C5O_CK2_A_1297 & 8.11 & 4.862343 & training \\
\hline 2C5O_CK2_C_1298 & 8.11 & 5.131049 & training \\
\hline 2EWA_SB2_A_361 & 15.98 & 13.587485 & training \\
\hline 2F2U_M77_A_501 & 14.07 & 10.874512 & training \\
\hline 2F2U_M77_B_1501 & 14.07 & 12.677324 & training \\
\hline 2J2I_LY4_B_1307 & 5.92 & 9.034196 & training \\
\hline 2NPQ_BOG_A_1000 & 10.39 & 10.77333 & training \\
\hline 2NPQ_BOG_A_2000 & 10.39 & 9.395628 & training \\
\hline 203P_QUE_A_501 & 15.21 & 11.99402 & training \\
\hline 2063_MYC_A_501 & 11.77 & 10.327488 & training \\
\hline 2064_MYU_A_501 & 12.6 & 15.132906 & training \\
\hline 2QHM_7CS_A_500 & 11.93 & 15.419877 & training \\
\hline 2RIO_ADP_A_1101 & 8.5 & 8.839855 & training \\
\hline 2RIO_ADP_B_2101 & 8.5 & 8.12286 & training \\
\hline 2RKU_R78_A_500 & 17.33 & 17.202715 & training \\
\hline 2UZT_SS3_A_1351 & 13.93 & 10.50961 & training \\
\hline 2VU3_LZE_A_1299 & 15.02 & 11.712693 & training \\
\hline
\end{tabular}


bioRxiv preprint doi: https://doi.org/10.1101/2020.09.28.316224; this version posted January 3, 2021. The copyright holder for this preprint (which was not certified by peer review) is the author/funder, who has granted bioRxiv a license to display the preprint in perpetuity. It is made available under aCC-BY-ND 4.0 International license.

\begin{tabular}{|c|c|c|c|}
\hline 2WTV_ZZL_A_1390 & 16.48 & 14.356284 & training \\
\hline 2WTV_ZZL_B_1390 & 16.48 & 14.618113 & training \\
\hline 2XJ2_985_A_1001 & 14.24 & 13.576728 & training \\
\hline 2Y7J_B49_A_1294 & 9.74 & 8.980873 & training \\
\hline 2Y7J_B49_B_1294 & 9.74 & 10.673288 & training \\
\hline 2Y7J_B49_C_1294 & 9.74 & 8.560607 & training \\
\hline 2Y7J_B49_D_1294 & 9.74 & 5.335432 & training \\
\hline 2YIW_YIW_A_1353 & 19.34 & 12.520471 & training \\
\hline 2YIX_YIX_A_1355 & 17.23 & 14.783937 & training \\
\hline 2ZB1_GK4_A_361 & 12.25 & 11.635828 & training \\
\hline 3AMA_SKE_A_351 & 9.22 & 6.7474203 & training \\
\hline 3AMB_VX6_A_351 & 9.22 & 11.450221 & training \\
\hline 3AT4_CCK_A_336 & 17.61 & 16.972866 & training \\
\hline 3BWJ_ARX_A_352 & 12.66 & 10.589522 & training \\
\hline 3D0E_G93_A_1 & 17.04 & 12.532833 & training \\
\hline 3D0E_G93_B_2 & 17.04 & 17.183178 & training \\
\hline 3DDQ_RRC_A_299 & 12.9 & 8.297859 & training \\
\hline 3DDQ_RRC_C_299 & 12.9 & 7.178257 & training \\
\hline 3E5A_VX6_A_500 & 17.73 & 12.699175 & training \\
\hline 3EQG_4BM_A_1 & 14.99 & 9.964328 & training \\
\hline 3FC1_52P_X_362 & 10.49 & 10.373807 & training \\
\hline 3FLS_FLS_A_361 & 18.24 & 13.88539 & training \\
\hline 3FLW_FLW_A_361 & 18.16 & 16.465137 & training \\
\hline 3FSK_RO6_A_450 & 14.96 & 13.170166 & training \\
\hline 3GCP_SB2_A_361 & 15.72 & 12.1016035 & training \\
\hline 3GCS_BAX_A_401 & 5.6 & 6.2856894 & training \\
\hline 3GCU_R48_B_401 & 13.32 & 12.8222475 & training \\
\hline 3GCV_SS6_A_361 & 14.12 & 12.847186 & training \\
\hline 3GNI_ATP_B_1 & 15.43 & 9.653784 & training \\
\hline 3GP0_NIL_A_1 & 14.84 & 18.384321 & training \\
\hline 3HEC_STI_A_1 & 9.22 & 8.287979 & training \\
\hline 3HEG_BAX_A_1 & 5.6 & 7.008605 & training \\
\hline 3HMO_STU_A_1 & 15.01 & 12.163776 & training \\
\hline 3HP5_52P_A_401 & 10.49 & 10.918285 & training \\
\hline 3HV6_R39_A_361 & 12.72 & 9.466206 & training \\
\hline 3IW5_DF3_A_362 & 11.69 & 9.562438 & training \\
\hline 3IW6_PP0_A_361 & 10.33 & 7.345064 & training \\
\hline 3IW8_HIZ_A_361 & 8.92 & 9.450232 & training \\
\hline 3JVS_AGY_A_900 & 13.44 & 14.485944 & training \\
\hline 3L8S_BFF_A_361 & 13.83 & 12.68417 & training \\
\hline 3L8X_N4D_A_361 & 16.12 & 14.4477 & training \\
\hline
\end{tabular}


bioRxiv preprint doi: https://doi.org/10.1101/2020.09.28.316224; this version posted January 3, 2021. The copyright holder for this preprint (which was not certified by peer review) is the author/funder, who has granted bioRxiv a license to display the preprint in perpetuity. It is made available under aCC-BY-ND 4.0 International license.

\begin{tabular}{|c|c|c|c|}
\hline |3LFA_1N1_A_361 & 8.22 & 9.860175 & training \\
\hline 3MYG_EML_A_1 & 22.34 & 17.777912 & training \\
\hline 3NPC_B96_A_365 & 9.53 & 10.554703 & training \\
\hline 3NPC_B96_B_365 & 9.53 & 8.5307665 & training \\
\hline 308P_BMU_A_361 & 11.34 & 10.370246 & training \\
\hline 308U_BMU_A_361 & 11.34 & 9.472248 & training \\
\hline 30BJ_BMU_A_361 & 11.34 & 11.657911 & training \\
\hline 3PG3_DG7_A_362 & 11.01 & 13.260027 & training \\
\hline 3PXF_2AN_A_304 & 7.91 & 8.43764 & training \\
\hline 3PXQ_2AN_A_300 & 7.91 & 8.458857 & training \\
\hline 3PXQ_2AN_A_301 & 7.91 & 7.9644737 & training \\
\hline 3PXQ_2AN_A_302 & 7.91 & 7.834199 & training \\
\hline 3PXZ_2AN_A_299 & 7.91 & 6.5781374 & training \\
\hline 3PXZ_JWS_A_301 & 9.74 & 7.590602 & training \\
\hline 3PY1_2AN_A_301 & 7.91 & 6.6630545 & training \\
\hline 3PY1_2AN_A_302 & 7.91 & 7.0281353 & training \\
\hline 3PY1_SU9_A_300 & 13.56 & 13.19573 & training \\
\hline 3RGF_BAX_A_465 & 5.78 & 8.478868 & training \\
\hline 3SW7_19K_A_299 & 9.76 & 12.033982 & training \\
\hline 3TZM_085_A_1 & 6.27 & 13.889732 & training \\
\hline 3UBD_SLO_A_400 & 10.45 & 11.020773 & training \\
\hline 3UO4_0C0_A_1 & 12.73 & 12.905641 & training \\
\hline 3UOL_0C7_A_2 & 15.54 & 13.293035 & training \\
\hline 3UOL_0C7_B_1 & 15.54 & 14.602809 & training \\
\hline 3VQH_IQB_A_401 & 15.29 & 15.187998 & training \\
\hline 3VVH_4BM_B_503 & 14.99 & 13.505534 & training \\
\hline 3VVH_4BM_C_503 & 14.99 & 15.487566 & training \\
\hline 3ZSH_469_A_400 & 19.12 & & training \\
\hline 3ZSI_52P_A_1000 & 10.49 & 7.871111 & training \\
\hline 4BCQ_TJF_C_1295 & 13.44 & 12.84407 & training \\
\hline 4BTK_DTQ_A_1337 & 12.95 & 9.336148 & training \\
\hline 4CRL_C1I_A_1360 & 20.06 & 15.915939 & training \\
\hline 4DLI_IRG_A_401 & 10.64 & 9.909659 & training \\
\hline 4DLI_IRG_A_402 & 10.64 & 11.62527 & training \\
\hline 4EK6_10K_A_301 & 9.92 & 10.227652 & training \\
\hline 4EZ7_2AN_A_302 & 7.91 & 7.93563 & training \\
\hline 4EZ7_2AN_A_303 & 7.91 & 6.879238 & training \\
\hline 4F9Y_GG5_A_401 & 12.03 & 14.077505 & training \\
\hline 4F9Y_GG5_A_402 & 12.03 & 12.441417 & training \\
\hline 4F9Y_LM3_A_403 & 13.21 & 10.422124 & training \\
\hline 4FKI_09K_A_301 & 7.45 & 10.119772 & training \\
\hline
\end{tabular}


bioRxiv preprint doi: https://doi.org/10.1101/2020.09.28.316224; this version posted January 3, 2021. The copyright holder for this preprint (which was not certified by peer review) is the author/funder, who has granted bioRxiv a license to display the preprint in perpetuity. It is made available under aCC-BY-ND 4.0 International license.

\begin{tabular}{|c|c|c|c|}
\hline 4FKL_CK2_A_300 & 8.11 & 7.9371223 & training \\
\hline 4FKO_20K_A_301 & 7.84 & 8.178918 & training \\
\hline 4FKU_60K_A_301 & 12.39 & 9.98849 & training \\
\hline 4FKU_60K_A_303 & 12.39 & 14.65224 & training \\
\hline 4GUE_QCT_A_401 & 9.76 & 10.720871 & training \\
\hline 4I3Z_ADP_A_301 & 0.67 & 5.0668316 & training \\
\hline 4I3Z_ADP_C_301 & 0.67 & 6.001342 & training \\
\hline 4I5M_R78_A_401 & 18.65 & 21.034546 & training \\
\hline 4JBQ_VX6_A_501 & 17.73 & 14.951972 & training \\
\hline 4KS8_B49_A_701 & 3.73 & 7.098278 & training \\
\hline 4L9I_8PR_A_601 & 8.95 & 12.205049 & training \\
\hline 4L9I_8PR_B_601 & 8.95 & 10.86388 & training \\
\hline 4LOO_SB4_A_401 & 16.48 & 16.590876 & training \\
\hline 4LOP_SB4_A_401 & 16.48 & 16.209667 & training \\
\hline 4LOP_SB4_B_401 & 16.48 & 14.673704 & training \\
\hline 4LOP_SB4_C_401 & 16.48 & 14.650061 & training \\
\hline 4LOP_SB4_D_401 & 16.48 & 14.583004 & training \\
\hline 4LOQ_SB4_A_401 & 16.48 & 15.305688 & training \\
\hline 4LOQ_SB4_B_401 & 16.48 & 12.504713 & training \\
\hline 4LOQ_SB4_C_401 & 16.48 & 13.820618 & training \\
\hline 4OTI_MI1_A_1001 & 13.13 & 12.5323515 & training \\
\hline 4QMN_DB8_A_401 & 11.62 & 14.84667 & training \\
\hline 4QMZ_B49_A_401 & 5.69 & 6.55925 & training \\
\hline 4QP2_36R_A_401 & 4.67 & 7.273883 & training \\
\hline 4QTA_38Z_A_411 & 15.86 & 13.754637 & training \\
\hline 4QTB_38Z_A_418 & 13.99 & 12.003158 & training \\
\hline 4QTB_38Z_B_412 & 13.99 & 11.510162 & training \\
\hline 4QTE_390_A_430 & 17.73 & 13.258313 & training \\
\hline 4QYY_3G7_A_401 & 14.51 & 10.219946 & training \\
\hline 4TXC_38G_A_301 & 12.95 & 11.588561 & training \\
\hline 4U43_3D8_A_401 & 7.65 & 5.3566923 & training \\
\hline 4X21_3WH_A_501 & 14.84 & 11.492774 & training \\
\hline 4X21_3WH_B_501 & 14.84 & 13.035936 & training \\
\hline 4XX9_RF4_A_402 & 9.44 & 8.770052 & training \\
\hline 4Y8D_49J_A_401 & 16.24 & 11.525299 & training \\
\hline 4Y8D_49J_B_401 & 16.24 & 15.099169 & training \\
\hline 4ZJI_4OQ_B_601 & 7.83 & 7.283536 & training \\
\hline 4ZJI_4OQ_C_601 & 7.83 & 9.089074 & training \\
\hline 4ZJI_4OQ_D_601 & 7.83 & 6.8545666 & training \\
\hline 4ZJJ_4OR_A_601 & 7.83 & 7.0933084 & training \\
\hline 4ZJJ_4OR_B_601 & 7.83 & 8.350812 & training \\
\hline
\end{tabular}


bioRxiv preprint doi: https://doi.org/10.1101/2020.09.28.316224; this version posted January 3, 2021. The copyright holder for this preprint (which was not certified by peer review) is the author/funder, who has granted bioRxiv a license to display the preprint in perpetuity. It is made available under aCC-BY-ND 4.0 International license.

\begin{tabular}{|c|c|c|c|}
\hline 4ZJJ_4OR_C_601 & 7.83 & 5.1687207 & training \\
\hline 4ZJJ_4OR_D_601 & 7.83 & 7.2560215 & training \\
\hline 5AJQ_DB8_A_800 & 16.48 & 14.70757 & training \\
\hline 5AJQ_DB8_B_800 & 16.48 & 14.7908 & training \\
\hline 5AUT_2AN_A_301 & 9.62 & 8.227689 & training \\
\hline 5CS6_K82_A_404 & 5.07 & 4.468193 & training \\
\hline 5CS6_K82_A_405 & 5.07 & 5.1155643 & training \\
\hline 5CS6_K82_A_406 & 5.07 & 4.588729 & training \\
\hline 5CS6_K82_A_407 & 5.07 & 4.937118 & training \\
\hline 5CSH_54E_A_401 & 5.92 & 5.2921076 & training \\
\hline 5CSH_54E_A_402 & 5.92 & 7.947792 & training \\
\hline 5CSH_54E_B_403 & 5.92 & 5.49813 & training \\
\hline 5CSH_54E_B_404 & 5.92 & 6.6865587 & training \\
\hline 5CSP_54G_A_401 & 7.46 & 6.192384 & training \\
\hline 5CU3_54S_A_404 & 12.66 & 16.073565 & training \\
\hline 5CU3_54S_B_403 & 12.66 & 9.69426 & training \\
\hline 5CU4_54S_A_404 & 12.66 & 12.544091 & training \\
\hline 5DN3_5DN_A_402 & 10.19 & 7.8209085 & training \\
\hline 5DR9_SKE_A_401 & 6 & 7.5168114 & training \\
\hline 5DRB_5FJ_A_501 & 17.11 & 14.781893 & training \\
\hline 5DT0_SKE_A_401 & 6 & 5.988515 & training \\
\hline 5JQ5_I74_A_302 & 10.67 & 13.649733 & training \\
\hline 5L4Q_LKB_A_401 & 14.46 & 12.602347 & training \\
\hline 5L4Q_LKB_B_401 & 14.46 & 14.451378 & training \\
\hline 5MO8_C98_A_404 & 11.02 & 11.826718 & training \\
\hline 5MO8_C98_B_401 & 11.02 & 11.957595 & training \\
\hline 5MOD_86L_A_404 & 5.81 & 5.3589926 & training \\
\hline 5MOE_OQC_A_409 & 5.3 & 4.6797047 & training \\
\hline 5MOE_OQC_A_410 & 5.3 & 5.384391 & training \\
\hline 5MOE_OQC_A_411 & 5.3 & 6.2392187 & training \\
\hline 5MOE_OQC_B_409 & 5.3 & 6.606739 & training \\
\hline 5MRB_C5N_A_901 & 12.27 & 16.349714 & training \\
\hline 5MTX_FJI_A_401 & 15.55 & 13.412746 & training \\
\hline 5MTY_HB9_A_401 & 18.16 & 13.793211 & training \\
\hline 5TBE_78L_A_401 & 16.94 & 19.41852 & training \\
\hline 5TE0_XIN_A_401 & 14.28 & 14.470296 & training \\
\hline 5TF9_7AV_A_501 & 10.04 & 7.720195 & training \\
\hline 5VC3_DB8_A_601 & 14.08 & 16.318983 & training \\
\hline 5VC4_XZN_A_601 & 14.65 & 10.742605 & training \\
\hline 5VC5_96M_A_601 & 16.03 & 12.171665 & training \\
\hline 5VC6_P48_A_601 & 15.82 & 14.553338 & training \\
\hline
\end{tabular}


bioRxiv preprint doi: https://doi.org/10.1101/2020.09.28.316224; this version posted January 3, 2021. The copyright holder for this preprint (which was not certified by peer review) is the author/funder, who has granted bioRxiv a license to display the preprint in perpetuity. It is made available under aCC-BY-ND 4.0 International license.

\begin{tabular}{|c|c|c|c|}
\hline 5VCV_1N1_A_404 & 15.72 & 9.767446 & training \\
\hline 5VCW_93J_A_401 & 4.51 & 5.9813185 & training \\
\hline 5VCW_93J_B_401 & 4.51 & 4.4235907 & training \\
\hline 5VCZ_XZN_A_401 & 12.33 & 13.311826 & training \\
\hline 5VD0_8X7_A_401 & 12.64 & 13.869585 & training \\
\hline 5VD1_P48_A_401 & 11.53 & 17.269943 & training \\
\hline 5VD3_H8H_A_401 & 10.48 & 12.441893 & training \\
\hline 1PY5_PY1_A_700 & 16.82 & 14.969334 & validation \\
\hline 1XH7_R96_A_351 & 13.42 & 14.521075 & validation \\
\hline 1XH9_R69_A_351 & 15.59 & 11.105695 & validation \\
\hline 2A4L_RRC_A_300 & 3.39 & 6.7256346 & validation \\
\hline 2FVD_LIA_A_299 & 19.12 & 15.857263 & validation \\
\hline 2UZW_SS4_E_1351 & 16.82 & 13.931561 & validation \\
\hline 2WTV_ZZL_D_1390 & 16.48 & 16.501717 & validation \\
\hline 3BWJ_ARX_A_351 & 12.66 & 10.008118 & validation \\
\hline 3GCQ_1BU_A_401 & 12.59 & 17.14584 & validation \\
\hline 3GCU_R48_A_401 & 13.32 & 11.107234 & validation \\
\hline 3GI3_B10_A_391 & 17.84 & 15.133845 & validation \\
\hline 3HMP_CX4_A_1 & 10.34 & 7.4939575 & validation \\
\hline 3HUB_469_A_361 & 19.12 & 14.843432 & validation \\
\hline 3HUC_G97_A_362 & 11.49 & 10.609373 & validation \\
\hline 3LFF_Z83_A_362 & 13.15 & 12.004912 & validation \\
\hline 308T_BMU_A_361 & 11.34 & 10.189346 & validation \\
\hline 3PXF_2AN_A_305 & 7.91 & 9.076647 & validation \\
\hline 3PXZ_2AN_A_300 & 7.91 & 6.3745623 & validation \\
\hline 3SW4_18K_A_299 & 9.95 & 14.080001 & validation \\
\hline 3U9N_09H_A_301 & 13.82 & 11.017376 & validation \\
\hline 3UVQ_FS8_A_361 & 15.59 & 11.264186 & validation \\
\hline 3VVH_4BM_A_703 & 14.99 & 12.857493 & validation \\
\hline 4BCQ_TJF_A_1296 & 13.44 & 12.502231 & validation \\
\hline 4BTJ_ATP_B_1338 & 8.3 & 10.083336 & validation \\
\hline 4KKH_1RQ_A_501 & 14.6 & 17.964321 & validation \\
\hline 4LOQ_SB4_D_401 & 16.48 & 22.440546 & validation \\
\hline 4NJ3_2KD_A_301 & 12.72 & 11.144922 & validation \\
\hline 4QMS_1N1_A_401 & 3.97 & 8.428536 & validation \\
\hline 4QMU_SKE_A_401 & 5.63 & 5.1778917 & validation \\
\hline 4ZJI_4OQ_A_601 & 7.83 & 5.5979853 & validation \\
\hline 5D1J_56H_A_4000 & 14.79 & 12.7819 & validation \\
\hline 5DPV_SKE_A_402 & 6 & 7.86001 & validation \\
\hline 5LVL_537_A_401 & 11.29 & 10.439565 & validation \\
\hline 5MOE_OQC_B_408 & 5.3 & 6.949047 & validation \\
\hline
\end{tabular}




\begin{tabular}{|l|l|l|l|}
\hline 5V5Y_8X7_A_601 & 15.83 & 15.258941 & validation \\
\hline 5VCY_DB8_A_401 & 12.83 & 14.903077 & validation \\
\hline 1KE9_LS5_A_299 & 10.95 & 10.35851 & test \\
\hline 1XH6_R94_A_351 & 14.55 & 14.093109 & test \\
\hline 2BAJ_1PP_A_401 & 17.04 & 12.874502 & test \\
\hline 2BAL_PQA_A_401 & 12.23 & 12.644074 & test \\
\hline 2WTV_ZZL_C_1392 & 16.48 & 13.081798 & test \\
\hline 2XJ1_XJ1_A_1307 & 15.09 & 12.160637 & test \\
\hline 3FLN_3FN_C_361 & 20.04 & 13.301807 & test \\
\hline 3HRF_P47_A_1374 & 9.19 & 9.049356 & test \\
\hline 3HV7_1AU_A_361 & 15.94 & 10.493131 & test \\
\hline 3LFE_Z84_A_361 & 12.05 & 12.960303 & test \\
\hline 3T11_B49_A_299 & 9.22 & 9.492598 & test \\
\hline 4EK8_16K_A_301 & 10.04 & 11.950938 & test \\
\hline 4FKP_LS5_A_301 & 10.95 & 11.706519 & test \\
\hline 4FKW_62K_A_301 & 13.95 & 14.523321 & test \\
\hline 5TCO_79Q_A_401 & 17.28 & 12.303295 & test \\
\hline 5TF9_7AV_B_501 & 10.04 & 10.4065275 & test \\
\hline
\end{tabular}

\section{Acknowledgements}

AA acknowledges funding awarded by the Indian Academy of Sciences, Bangalore (2019).

BM would like to acknowledge Tata Trusts-TDU Fellowship for PhD awarded to her from 2017 to 2019. All authors acknowledge NCBS for infrastructural support.

\section{Conflict of interest}

The authors declare no conflict of interest.

\section{Author Contributions}

Conceptualization: Bhavika Mam, Asad Ahmed

Data curation: Asad Ahmed, Bhavika Mam

Formal analysis: Asad Ahmed, Bhavika Mam

Funding acquisition: Ramanathan Sowdhamini.

Investigation: Asad Ahmed, Bhavika Mam

Methodology: Asad Ahmed, Bhavika Mam

Project administration: Ramanathan Sowdhamini

Resources: Ramanathan Sowdhamini

Supervision: Ramanathan Sowdhamini

Validation: Asad Ahmed, Bhavika Mam

Visualization: Asad Ahmed

Writing - Asad Ahmed, Bhavika Mam 
Writing - review \& editing: Ramanathan Sowdhamini

\section{Author ORCIDs}

1. Asad Ahmed

2. Bhavika Mam

3. Prof. Ramanathan Sowdhamini
: https://orcid.org/0000-0003-3775-9320

: https://orcid.org/0000-0002-3130-0925

: https://orcid.org/0000-0002-6642-2367 